\title{
Stimulated quasiparticles in spin-split superconductors
}

\author{
P. Virtanen, ${ }^{1,2}$ T. T. Heikkilä, ${ }^{2}$ and F. S. Bergeret ${ }^{3,4}$ \\ ${ }^{1}$ Low Temperature Laboratory, Department of Applied Physics, P.O. Box 15100, FI-00076 Aalto University, Finland \\ ${ }^{2}$ University of Jyvaskyla, Department of Physics and Nanoscience Center, P.O. Box 35, FI-40014 University of Jyväskylä, Finland \\ ${ }^{3}$ Centro de Física de Materiales (CFM-MPC), Centro Mixto CSIC-UPV/EHU, Manuel de Lardizabal 5, E-20018 San Sebastián, Spain \\ ${ }^{4}$ Donostia International Physics Center (DIPC), Manuel de Lardizabal 5, E-20018 San Sebastián, Spain
}

(Received 10 November 2015; published 19 January 2016)

\begin{abstract}
In superconductors spin split by an exchange field, thermal effects are coupled to spin transport. We show how an oscillating electromagnetic field in such systems creates spin imbalance, that can be detected with a spin-polarized probe. The sign and magnitude of the probe signal result from a competition between processes converting field-induced spin energy imbalance to spin imbalance, dominant at low frequencies, and microwave-driven pair breaking at high frequencies. In the presence of spin-flip scattering, we show that ac excitation also leads to multistabilities in the superconducting state.
\end{abstract}

DOI: 10.1103/PhysRevB.93.014512

\section{INTRODUCTION}

Long-lived spin excitations are interesting for spintronics applications, and the spin transport in superconductors has recently attracted renewed attention in this context [1-3]. Spin accumulation in a superconductor can be generated by injecting current from a spin-polarized electrode, for example, a ferromagnet. A second approach for spin injection studied in a number of recent experiments [4-9] is to use a magnetic field or proximity to ferromagnetic insulators to Zeeman split the density of states of the superconductor [10,11] (cf. Fig. 1), so that injection of current from an unpolarized probe also generates observable spin accumulation. The spin splitting also changes the quasiparticle physics so that one component of the imbalance only relaxes via inelastic scattering [4,5,12-14], leading to long observed spin lifetimes and relaxation lengths.

The physics of the long-ranged quasiparticle spin accumulation in spin-split superconductors is closely connected to their thermoelectric properties. Magnetic interactions in superconductors break the spin-resolved electron-hole symmetry, enabling large thermoelectric responses. This is predicted to occur due to magnetic impurities [15], spin-active interfaces [16-18], and in superconductor-ferromagnet systems in the presence of exchange fields [19]. The large thermoelectric effect in spin-split superconductor/ferromagnet tunnel junction has been observed very recently (see Ref. [20]).

The thermoelectric mechanisms are also connected to photoelectric effects in superconductors [21,22], where absorbed radiation is converted to a dc voltage observed in a probe electrode. Based on the above discussion, a photo-spin-electric effect should be present also in spin-split superconductorsthe absorbed radiation generates spin imbalance, which relaxes slowly via inelastic scattering. This is interesting to consider, e.g., in the context of measurements that use microwave signals to probe spin resonances of the quasiparticles [9].

In this work, we discuss how an electric ac field in diffusive spin-split superconductors produces spin imbalance [see Fig. 1(a)]. We find that the ac driving generates spin imbalance that is either parallel or antiparallel to the exchange field, depending on the drive frequency. Nonequilibrium states generated by ac fields in conventional superconductors have long been studied [23], and we extend the picture to include spin splitting. Although interaction with the fields conserves spin, combining it with elastic spin-flip scattering from, e.g., magnetic impurities results to a nonequilibrium steady state with nonzero spin imbalance [see Fig. 1(b)]. We discuss how the effect can be detected via ferromagnetic probes [Eq. (13)]. Similar photoelectric effects are known to occur also in the absence of spin splitting [21,22], but they require weak elastic scattering. We also predict that the spin imbalance results to an instability in the superconducting order parameter, permitting multiple nonzero values for it in a temperature range around $T_{c}$, leading to hysteresis and providing a second characteristic signature of the effect.

The manuscript is organized as follows. We outline the model in Sec. II and discuss observables accessible with spinpolarized electrical probes in Sec. III. Modification of the superconducting order parameter is discussed in Sec. IV, and we conclude in Sec. V.

\section{KINETIC EQUATIONS}

We consider a diffusive superconductor film with a Zeeman field induced either by an external magnetic field [10] or, for example, proximity to a magnetic insulator $[11,24]$. In order to describe a nonequilibrium situation we apply the quasiclassical Keldysh-Green function formulation [25-29], and write the Usadel equation for the spin-split superconductor (here and below, we set $\left.\hbar=e=k_{B}=1\right)$ :

$$
D \hat{\nabla} \cdot(\check{g} \hat{\nabla} \check{g})+\left[i \epsilon \hat{\tau}_{3}-i(\boldsymbol{h} \cdot \boldsymbol{S}) \hat{\tau}_{3}-\hat{\Delta}-i \check{\sigma}, \check{g}\right]=0 .
$$

The function $\check{g}\left(t, t^{\prime}\right)$ is a matrix which in the Keldysh-Nambuspin space has the form,

$$
\check{g}=\left(\begin{array}{cc}
\hat{g}^{R} & \hat{g}^{K} \\
0 & \hat{g}^{A}
\end{array}\right),
$$

where $\hat{g}^{R, A, K}$ are the retarded, advanced, and Keldysh $2 \times 2$ matrices in the Nambu $\left(\tau_{j}\right)$ and spin $\left(s_{j}\right)$ spaces and $\boldsymbol{S}=$ $\left(s_{1}, s_{2}, s_{3}\right)$. The exchange field $\boldsymbol{h}$ is induced by an external magnetic field [10] or, for example, proximity to a magnetic insulator $[11,24]$. Here, $D$ is the diffusion constant of the superconductor, $\Delta$ is the order parameter, and $\check{\sigma}$ a self-energy corresponding to spin-flip and inelastic scattering (electronphonon or electron-electron). We use a gauge where the 
(a)

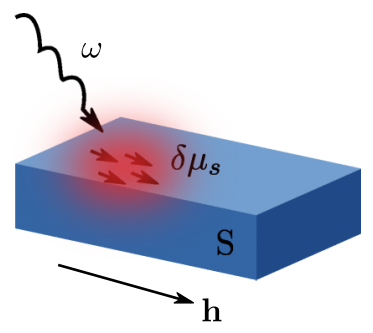

(b)

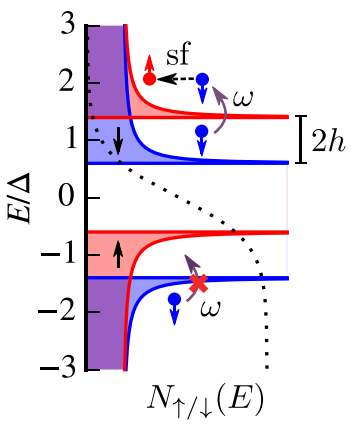

FIG. 1. (a) A superconductor absorbs microwave electromagnetic radiation, in the presence of an internal exchange field $\boldsymbol{h}$. In the steady state, this generates a spin imbalance $\delta \mu_{s}$, an excess of quasiparticles whose spins are either aligned $\left(\delta \mu_{s}>0\right)$ or antialigned $\left(\delta \mu_{s}<0\right)$ with the axis of the exchange field. (b) The quasiparticle spectrum is spin split by the exchange field. Coupling to microwaves generates spin-conserving quasiparticle transitions that change energy by $\pm \omega$ and perturb the electron distribution (dotted). Elastic spin-flip scattering transforms quasiparticles to the opposite spin species, converting energy imbalance to spin imbalance.

electric potential is $\varphi=0$, and coupling to electromagnetic fields is via a vector potential $\boldsymbol{A}$ appearing in the covariant derivative $\hat{\nabla} X=\nabla X-\left[i \boldsymbol{A} \tau_{3}, X\right]$.

We assume that the superconducting film is in a uniform time-dependent electric field $\mathcal{E}(t)=A_{0} \omega_{0} \sin \left(\omega_{0} t\right)$. We follow a similar approximation procedure as in Ref. [23]. In a spatially uniform situation, assuming the film is thinner than the skin depth, the vector potential enters equivalently as a self-energy $\check{\sigma}_{A}\left(t, t^{\prime}\right)=-i D \boldsymbol{A}(t) \cdot \hat{\tau}_{3} \check{g}\left(t, t^{\prime}\right) \boldsymbol{A}\left(t^{\prime}\right) \hat{\tau}_{3}$ which after time averaging is given by

$$
\check{\sigma}_{A}(E)=-i \frac{D A_{0}^{2}}{4} \tau_{3}\left[\check{g}\left(E+\omega_{0}\right)+\check{g}\left(E-\omega_{0}\right)\right] \tau_{3} .
$$

Considering time dynamics implied by Eq. (1), this expression describes the effect of the ac field in the leading order in the small parameter $D A_{0}^{2} \ll \omega_{0}$.

The self-energy term in Eq. (1) also takes into account a number of relaxation processes present in real superconductors. This includes spin-flip scattering [25,28,29] due to magnetic impurities $\check{\sigma}_{\text {sf }}=-\frac{i}{8 \tau_{\mathrm{sf}}} \boldsymbol{S} \tau_{3} \cdot \check{g} \boldsymbol{S} \tau_{3}$, spin-orbit scattering $\check{\sigma}_{\text {so }}=-\frac{i}{8 \tau_{\mathrm{so}}} \boldsymbol{S} \cdot \check{g} \boldsymbol{S}$, and phonon scattering $\check{\sigma}_{\mathrm{ph}}$ (see Appendix A). Below, we parametrize $\tau_{\text {sf } / \text { so }}^{-1}=\frac{1 \pm \beta}{2} \tau_{\mathrm{sn}}^{-1}$, where the parameter $-1 \leqslant \beta \leqslant 1$ describes which of the spin-flip and spin-orbit scattering mechanisms is stronger. For example, the scattering rates in $\mathrm{Al}$ wires were found to be $\tau_{\mathrm{sn}} \approx 100 \mathrm{ps}$ and $\beta \approx 0.5$ in Ref. [30], so that $T_{c} \tau_{\mathrm{sn}} \sim 20$. We also include orbital dephasing, $\check{\sigma}_{\text {orb }}=-\frac{i}{2 \tau_{\text {orb }}} \tau_{3} \check{g} \tau_{3}$, which is relevant if an external magnetic field is used to generate the exchange field $h=g \mu_{B} B$. The scattering rate associated with the orbital effect is [31] $\tau_{\text {orb }}^{-1}=\frac{T_{c 0} \alpha_{\text {orb }}}{2}\left(h / T_{c 0}\right)^{2}$, where $T_{c 0} \approx 0.567 \Delta_{0}$ is the BCS critical temperature, and the parameter $\alpha_{\text {orb }}=$ $T_{c 0} D W^{2} /\left(12 g^{2} \mu_{B}^{2}\right)$ depends on the film thickness $W$.

The Keldysh component of $\breve{g}$ can be expressed in terms of the retarded and advanced matrices and distribution function matrix $\hat{f}, \hat{g}^{K}=\hat{g}^{R} \hat{f}-\hat{f} \hat{g}^{A}$. In particular $\hat{f}$ parametrizes the quasiparticle nonequilibrium modes. Below,

we choose the $z$ axis parallel to the Zeeman field. In this case, the retarded function is spin diagonal and we write it in the form $\hat{g}^{R}=\sum_{\sigma=\uparrow / \downarrow} s_{\sigma}\left[g_{\sigma, 1} \tau_{1}+g_{\sigma, 3} \tau_{3}\right]$, where $s_{\uparrow / \downarrow}=\left[1 \pm s_{z}\right] / 2$. Similarly, we write the distribution function as $\hat{f}=\sum_{\sigma=\uparrow / \downarrow} s_{\sigma}\left[f_{L \sigma}+\hat{\tau}_{3} f_{T \sigma}\right]$. The distribution functions $f_{T \sigma}$ characterize the charge imbalance and $f_{L \sigma}$ the energy imbalance in the two spin bands. An alternative representation $f_{T / L}=\left(f_{T / L, \uparrow}+f_{T / L, \downarrow}\right) / 2, f_{L 3 / T 3}=\left(f_{T / L, \uparrow}-\right.$ $\left.f_{T / L, \downarrow}\right) / 2$ was used in Ref. [12].

In terms of these functions, Eq. (1) results in kinetic equations for the components of $\hat{f}$. In the steady state they are rate equations expressing a balance of excitation and relaxation processes:

$$
\hat{\mathcal{I}}_{A}[\hat{f}]+\hat{\mathcal{I}}_{\text {sf }+\mathrm{so}}[\hat{f}]+\hat{\mathcal{I}}_{\Delta}[\hat{f}]+\hat{\mathcal{I}}_{\text {relax }}[\hat{f}]=0,
$$

where the collision integrals $\hat{\mathcal{I}}$ are related to the corresponding self-energies and $\hat{\Delta}$ via $\hat{\mathcal{I}}=\left(\hat{g}^{R} \hat{Z}-\hat{Z} \hat{g}^{A}\right) / 8, \hat{Z}=i \hat{\sigma}^{R} \hat{f}-$ $i \hat{f} \hat{\sigma}^{A}-i \hat{\sigma}^{K}$. Below, we find that $f_{T \sigma}=0$ for our problem, so that $\mathcal{I}_{\Delta}=0$.

For the electromagnetic collision integral we get

$$
\hat{Z}_{A}=\frac{D A_{0}^{2}}{4} \sum_{ \pm} \tau_{3}\left[g_{ \pm}^{R}\left(\hat{f}-\hat{f}_{ \pm}\right)-\left(\hat{f}-\hat{f}_{ \pm}\right) g_{ \pm}^{A}\right] \tau_{3},
$$

where $\hat{f}_{ \pm}(E)=\hat{f}\left(E \pm \omega_{0}\right)$. The $s_{\uparrow / \downarrow}$ components read

$$
\begin{aligned}
\mathcal{I}_{A, \sigma}(E)= & \operatorname{Tr} s_{\sigma} \hat{\mathcal{I}}=\frac{D A_{0}^{2}}{4} \sum_{ \pm} R_{L, \sigma}(E, E \pm \omega) \\
& \times\left[f_{L, \sigma}(E)-f_{L, \sigma}(E \pm \omega)\right] .
\end{aligned}
$$

The $\hat{\tau}_{3} s_{\sigma}$ components vanish, reflecting charge conservation. The $\pm \omega$ terms indicate driven quasiparticle transitions up/down in energy. In terms of the Fermi distribution function $f_{\sigma}=\frac{1-f_{L \sigma}}{2}$, the second line acquires the typical structure for fermion transitions, $-2\left\{f_{\sigma}(E \pm \omega)\left[1-f_{\sigma}(E)\right]-f_{\sigma}(E)[1-\right.$ $\left.\left.f_{\sigma}(E \pm \omega)\right]\right\}$. The kernel $R$ is

$$
R_{L, \sigma}\left(E, E^{\prime}\right)=N_{\sigma}(E) N_{\sigma}\left(E^{\prime}\right)+\operatorname{Im} g_{\sigma, 1, E} \operatorname{Im} g_{\sigma, 1, E^{\prime}},
$$

where $N_{\sigma}(E)=\operatorname{Re} g_{\sigma, 3, E}$ is the spin-dependent density of states. The result Eq. (4) is equivalent to a standard photoabsorption collision integral for each spin.

For the elastic spin-flip and spin-orbit scattering, we have

$$
\begin{gathered}
\mathcal{I}_{\mathrm{sn}, \sigma}=\frac{S_{\uparrow \downarrow}}{4 \tau_{\mathrm{sn}}}\left(f_{L, \sigma}-f_{L,-\sigma}\right), \\
S_{\uparrow \downarrow}=N_{\uparrow} N_{\downarrow}+\beta \operatorname{Im} g_{\uparrow, 1} \operatorname{Im} g_{\downarrow} .,
\end{gathered}
$$

To find analytical results we describe inelastic relaxation within a relaxation-time approximation, for which we have

$$
\mathcal{I}_{\text {in }, \sigma}=\frac{N_{\sigma}}{2 \tau_{\text {in }}}\left(f_{L \sigma}-f_{L}^{(0)}\right) .
$$

We also obtain numerical results with a more detailed model for electron-phonon scattering (see Appendix A).

Based on the above equations, we can first solve the components of $\hat{g}^{R}$ from Eq. (1), which can be done analytically in some cases, or in general numerically. This provides the coefficients in the kinetic equations (2). Alternatively, we also solve Eq. (1) directly numerically (see Appendix B), which ensures self-consistency of the spectral functions. 


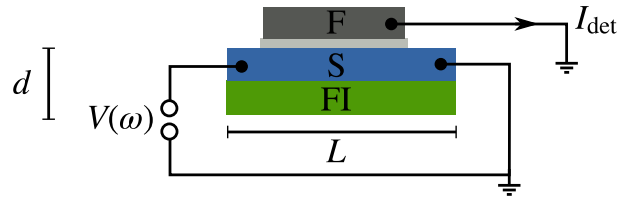

FIG. 2. Schematic cross section of a superconductor (S) / ferromagnetic insulator (FI) hybrid thin-film structure of thickness $d \ll L$, driven by an oscillating ac electric field corresponding to voltage $V$ at frequency $\omega$. The S/FI layer is coupled to a ferromagnetic detector probe $(\mathrm{F})$ via a tunnel junction, where the dc current $I_{\text {det }}$ is measured.

\section{SPIN IMBALANCE}

Experimentally, spin imbalance in the superconductor can be probed by electrical measurements that use spin-filtering probe junctions, for example, ferromagnets. The dc currentvoltage relation will in these cases contain a component that depends on the polarization of the probe and the spin imbalance in the sample.

The current measured by a spin-filtering tunnel probe (see Fig. 2) coupled to the superconductor and biased at $V=0$ is given by [32]

$$
\begin{gathered}
R_{\operatorname{det}} I_{\operatorname{det}}=\mu+P_{\operatorname{det}} \mu_{z} \\
\mu=\frac{1}{4} \int_{-\infty}^{\infty} d \epsilon\left[N_{\uparrow} f_{T \uparrow}+N_{\downarrow} f_{T \downarrow}\right], \\
\mu_{z}=\frac{1}{4} \int_{-\infty}^{\infty} d \epsilon\left\{N_{\uparrow}\left[f_{L \uparrow}-f_{L, d}\right]-N_{\downarrow}\left[f_{L \downarrow}-f_{L, d}\right]\right\},
\end{gathered}
$$

where $P_{\text {det }}$ is the detector polarization in the $z$ direction, $R_{\operatorname{det}}$ the junction resistance, and $f_{L, d}=\tanh \frac{E}{2 T_{\text {det }}}$ the equilibrium distribution in the detector. The current in the detector is a measure of the charge $(\mu)$ and spin $\left(\mu_{z}\right)$ imbalances.

The ac drive only excites the modes $f_{L, \uparrow / \downarrow}$ which carry no charge imbalance, so that on this level of analysis, no photoelectric effect is present $(\mu=0)$. Moreover, Eq. (2) together with a spin-independent relaxation time yields no spin imbalance $\left(\mu_{z}=0\right)$. This follows from $R_{L, \sigma}\left(E, E^{\prime}\right)=R_{L, \sigma}\left(E^{\prime}, E\right)$ and $\mu_{z} \propto \int_{-\infty}^{\infty} d E \sum_{\sigma} \sigma \mathcal{I}_{\text {in }, \sigma} \propto \int_{-\infty}^{\infty} d E \sum_{\sigma} \sigma \mathcal{I}_{A, \sigma}(E)=0$, reflecting conservation of spin.

In practice, however, elastic spin-flip scattering cannot be ignored, and the associated scattering times can be short compared to the inelastic collisions, $\tau_{\text {sn }} \ll \tau_{\text {in }}$. Under such conditions, ac drive can result to nonzero spin imbalance $\mu_{z} \neq 0$ in the steady state. Away from the strict diffusive limit, magnetic impurities result to a photoelectric effect of order $\ell_{\mathrm{el}}^{2} A_{0}^{2}$ also in the absence of the Zeeman splitting [21,22]. Here, we concentrate only on the diffusive limit and hence the Zeeman splitting is crucial.

Writing the solution of the kinetic equations [Eqs. (2) and (9)] using the relaxation time approximation for inelastic processes, and considering the limit of weak spin-flip scattering $\tau_{\text {in }} \ll \tau_{\text {sn }}, D A_{0}^{2} \tau_{\text {in }} \ll 1$, we find

$$
f_{L \sigma}-f_{L \sigma}^{(0)} \simeq-\frac{2 \mathcal{I}_{A, \sigma}^{(0)} \tau_{\mathrm{in}}}{N_{\sigma}}-\sigma \frac{S_{\uparrow \downarrow} \tau_{\mathrm{in}}^{2}}{2 \tau_{\mathrm{sn}}} \frac{N_{\uparrow} \mathcal{I}_{A \downarrow}^{(0)}-N_{\downarrow} \mathcal{I}_{A \uparrow}^{(0)}}{N_{\uparrow} N_{\downarrow}},
$$

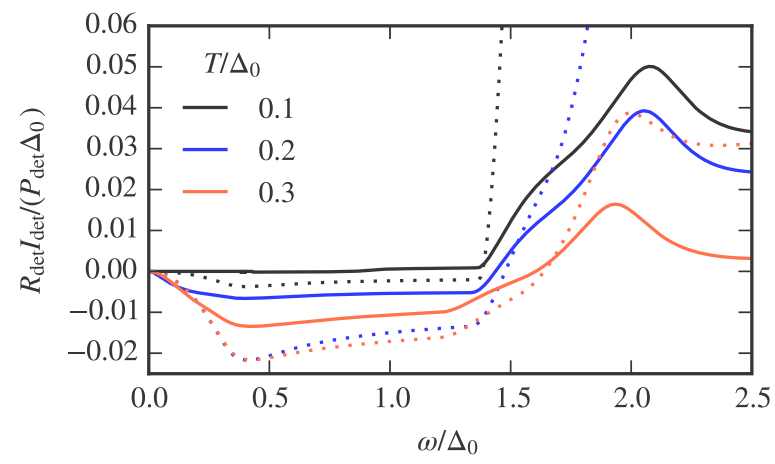

FIG. 3. Current at the detector probe under ac excitation, for $\tau_{\text {sn }} T_{c 0}=12.5, \beta=0.5, h / \Delta_{0}=0.17, D A_{0}^{2} / \Delta_{0}=4 \times 10^{-4}, \alpha_{\text {orb }}=$ 0.1 . Solid lines correspond to a numerical solution of Eq. (1) with the phonon model with $\tau_{\mathrm{eph}, 0} T_{c 0}=100$, and dotted lines to Eq. (13) with $\tau_{\text {in }}=\left(T_{c 0} / T\right)^{3} \tau_{\text {eph }, 0}$.

where $\mathcal{I}_{A \sigma}^{(0)}=\mathcal{I}_{A \sigma}\left[f_{L}^{(0)}\right]$, and $f_{L}^{(0)}=\tanh \frac{E}{2 T}$ is the equilibrium distribution. As noted above, the first term does not contribute to spin imbalance, but the second term does. The result is illustrated in Fig. 1(b): The transitions driven by the ac field generate an imbalance of quasiparticles inside both spin bands, which the spin-flip scattering converts to a spin imbalance at energies $|E|>|\Delta|+|h|$.

In a typical situation, however, we expect that spin-flip scattering is fast compared to inelastic relaxation $\left(\tau_{\mathrm{sn}} \ll \tau_{\text {in }}\right)$. In this limit we have for the detector current,

$$
\begin{aligned}
I_{\mathrm{det}}= & -\frac{P_{\mathrm{det}} D A_{0}^{2} \tau_{\text {in }}}{4 R_{\mathrm{det}}} \int_{-\infty}^{\infty} d E \sum_{ \pm}\left[f_{L}^{(0)}(E)-f_{L}^{(0)}(E \pm \omega)\right] \\
& \times \frac{N_{\uparrow}(E) R_{L \downarrow}(E, E \pm \omega)-N_{\downarrow}(E) R_{L \uparrow}(E, E \pm \omega)}{N_{\uparrow}(E)+N_{\downarrow}(E)} .
\end{aligned}
$$

The result is shown in Fig. 3 for representative parameters. The figure also shows results computed numerically using a phonon model (see Appendix A). The two are qualitatively similar, up to differences mainly originated from temperature and energy dependence in the relaxation rates. As the frequency increases, the amplitude of the signal also increases up to the point $\omega \approx 2 h$, where the process depicted in Fig. 1(b) saturates. At large frequencies $\omega \gtrsim 2 \Delta$ the detector current changes sign, as microwave-driven pair breaking starts to contribute. In this regime, the resulting signal can be understood as a thermoelectric current $[16,18,19]$ driven by a temperature difference $T_{S}>T_{F}$ caused by the heating of the superconductor by the drive.

In addition to the nonequilibrium-generated signal, oscillating electric fields can introduce a voltage drop $V_{\text {ac }}$ across the detector tunnel barrier. This results to an additional signal from photoassisted tunneling. Within the above approach, the total current is given by the Tien-Gordon result [33,34],

$$
I=\sum_{n=-\infty}^{\infty} J_{n}^{2}\left(\frac{e V_{\mathrm{ac}}}{\hbar \omega_{\mathrm{ac}}}\right) I_{\mathrm{det}}\left(V_{\mathrm{dc}}+n \hbar \omega_{\mathrm{ac}} / e\right),
$$


where $I_{\text {det }}(V)$ is the current-voltage relation in the absence of $V_{\text {ac }}$, and $J_{n}$ are Bessel functions. In the leading order in driving amplitude $\left(V_{\mathrm{ac}} \rightarrow 0\right)$ and without dc bias $(V=0)$,

$$
\begin{gathered}
I \simeq I_{\mathrm{det}}(0)+I_{0, T}, \\
I_{0, T}=\frac{V_{\mathrm{ac}}^{2}}{4 \omega^{2}}\left[I_{\mathrm{eq}}(\omega)+I_{\mathrm{eq}}(-\omega)\right],
\end{gathered}
$$

where $I_{\mathrm{det}}(0)$ is the nonequilibrium current (9), $I_{0, T}$ the photoassisted current, and $I_{\mathrm{eq}}(V)$ is the IV relation of the FIS junction when the superconductor is at equilibrium. We have

$$
\begin{aligned}
I_{0, T}= & \frac{P_{\mathrm{det}} V_{\mathrm{ac}}^{2}}{2 R_{\mathrm{det}} \omega^{2}} \int_{-\infty}^{\infty} d E N_{0}(E)\left[f_{L}^{(0)}(E+h)\right. \\
& -\frac{f_{L}^{(0)}(E-\omega+h)+f_{L}^{(0)}(E+\omega+h)}{2} \\
& \left.-f_{L}^{(0)}(E-h)+\frac{f_{L}^{(0)}(E-\omega-h)+f_{L}^{(0)}(E+\omega-h)}{2}\right],
\end{aligned}
$$

where $N_{0}=\operatorname{Re} \frac{|E|}{\sqrt{E^{2}-\Delta^{2}}}$ is the BCS density of states. Consider now $\omega, h \lesssim T$. We find

$$
I_{0, T}=-\frac{P_{\mathrm{det}} V_{\mathrm{ac}}^{2} h}{2 R_{\mathrm{det}} \Delta_{0}^{2}} \eta(T / \Delta),
$$

where $\eta \sim 1$. The photoassisted tunneling current has the same sign $I<0$ as a thermoelectric current generated by heating the ferromagnet, $T_{S}<T_{F}$, and therefore also the same sign as the nonequilibrium effect (13) at the low frequencies. The ratio of the photoassisted tunneling to the nonequilibrium one is $I_{\mathrm{det}}(0) / I_{0, T} \propto\left(A_{0} \omega_{0} \ell_{\text {in }} / V_{\mathrm{ac}}\right)^{2}$ where $\ell_{\text {in }}=\sqrt{D \tau_{\text {in }}}$.

In principle, $V_{\text {ac }}$ can be suppressed by suitable circuit design. An approach for suppressing the photoassisted tunneling used in a previous experiment [35] measuring the gap enhancement due to microwave drive was to use a large-area lateral tunnel junction with a high capacitance. Assuming the microwave currents in the $S$ film and through the detector junction are of similar magnitude (cf. Ref. [36]), one has $A_{0} \omega_{0} \ell_{\text {in }} / V_{\text {ac }} \propto Z_{S, \ell_{\text {in }}}(\omega) / Z_{T}(\omega)$, where $Z_{S, \ell_{\text {in }}}$ is the impedance of the $S$ film of length $\ell_{\text {in }}$ and $Z_{T}$ is the junction impedance. Moreover, the presence of photoassisted tunneling can in principle be recognized from the appearance of frequency replicas in the tunneling I-V relation, which should not be present in the nonequilibrium signal.

\section{GAP INSTABILITY}

The nonequilibrium spin accumulation affects the magnitude of the superconducting order parameter, potentially leading to large changes and collapse of superconductivity for large driving amplitudes.

Let us consider the effect of the driving on the superconducting order parameter,

$$
\Delta=\frac{\lambda}{8} \int_{-E_{0}}^{E_{0}} d E \operatorname{tr} \frac{\tau_{1}+i \tau_{2}}{2} \hat{g}^{K}(E) .
$$

Here, we assume singlet pairing, and $\lambda$ is the corresponding coupling constant and $E_{0}$ the BCS cutoff. The simplest situation is obtained by neglecting spin-flip and spin-orbit scattering. In this case, we can observe that the only spin structure in the equations arises from the Zeeman term, $\boldsymbol{h} \cdot \boldsymbol{S}$. Treating inelastic collisions within a relaxation time approximation, we find in leading order,

$$
\begin{aligned}
\frac{N_{\sigma} \delta f_{L \sigma}}{\tau_{\text {in }}}= & -\frac{D A_{0}^{2}}{2} \sum_{ \pm} R_{L \sigma}(E, E \pm \omega) \\
& \times\left[f_{L}^{(0)}(E)-f_{L}^{(0)}(E \pm \omega)\right] .
\end{aligned}
$$

The nonequilibrium part of the gap equation now reads

$$
\begin{aligned}
\delta \Delta= & \frac{\lambda}{4} D A_{0}^{2} \tau_{\text {in }} \int_{-\infty}^{\infty} d E \sum_{\sigma= \pm} \sum_{ \pm} \frac{R_{ \pm}^{(0)}(E) F_{0}(E)}{N_{0}(E)} \\
& \times\left[f_{0}(E+\sigma h)-f_{0}(E+\sigma h \pm \omega)\right] \\
\simeq & \frac{\lambda}{4} \frac{D A_{0}^{2} \tau_{\text {in }} \omega}{2 T} \int_{-\infty}^{\infty} d E \sum_{ \pm} \frac{ \pm R_{ \pm}^{(0)}(E) F_{0}(E)}{N_{0}(E)},
\end{aligned}
$$

where on the second line we expand around $T \gg h, \Delta$, and $F_{0}(E)=\left.\frac{1}{4} \operatorname{Im} \operatorname{tr} \tau_{1} \hat{g}^{R}(E)\right|_{h=0}$. The result is the same as for zero Zeeman field. Without spin-flip scattering, the exchange field does not have a significant effect at high temperatures, and the result coincides with known results in Ref. [23]: The superconducting gap is enhanced by the driving, and the superconducting branch extends to $T>T_{c}$. Numerical calculations also indicate that the exchange field does not cause significant qualitative changes at lower temperatures either (see below).

The spin imbalance generated by the spin-flip scattering, however, modifies the above conclusion, provided these processes are not slow compared to energy relaxation. As above, let us now assume $\tau_{\mathrm{sn}} \ll \tau_{\text {in }}$. In this case we find

$$
\begin{aligned}
\delta \Delta= & \frac{\lambda}{4} D A_{0}^{2} \tau_{\text {in }} \int_{-\infty}^{\infty} d \epsilon \frac{F_{\uparrow}+F_{\downarrow}}{N_{\uparrow}+N_{\downarrow}} \\
& \times \sum_{ \pm} R_{L}(E, E \pm \omega)\left[f_{L}^{(0)}(E)-f_{L}^{(0)}(E \pm \omega)\right],
\end{aligned}
$$

where $R_{L}=\frac{1}{2} \sum_{\sigma} R_{L \sigma}$, and $F_{\sigma}=\operatorname{Re} g_{\sigma, 1}$ is the coherence function. The difference to Eq. (21) is in that the elastic spinflip scattering forces the quasiparticle distributions for both spins to be the same, rather than being copies of a single distribution shifted by the exchange field.

In order to obtain analytical results, let us consider a situation in which the effect of scattering on the spectral functions is small, $1 / \Delta \ll \tau_{\mathrm{sf}}, \tau_{\mathrm{so}}, \tau_{\text {orb }}$. Then, $N_{\sigma}(E)=$ $N(E-\sigma h), F_{\sigma}(E)=F(E-\sigma h)$, where $N(E)=N(-E)=$ $\operatorname{Re}\left[E / \sqrt{E^{2}-\Delta^{2}}\right]$ is the BCS density of states, and $F(E)=$ $-F(-E)=\operatorname{Re}\left[\Delta / \sqrt{E^{2}-\Delta^{2}}\right]$ the BCS coherence function. Close to the critical temperature $T \approx T_{c}(h)$ and neglecting the orbital effect, the gap equation can be expanded to the form $[27,37,38]$,

$$
\ln \frac{T_{c}}{T}=\frac{7 \zeta(3)-186 \zeta(5) \frac{h^{2}}{4 \pi^{2} T^{2}}}{8 \pi^{2} T^{2}} \Delta^{2}-\frac{D A_{0}^{2} \tau_{\text {in }} \omega}{4 T} P\left(\frac{\omega}{\Delta}, \frac{h}{\Delta}\right) .
$$

The nonequlibrium part $\delta \Delta$ results to an extra term [23,27], whose parameter dependence is given by the dimensionless 


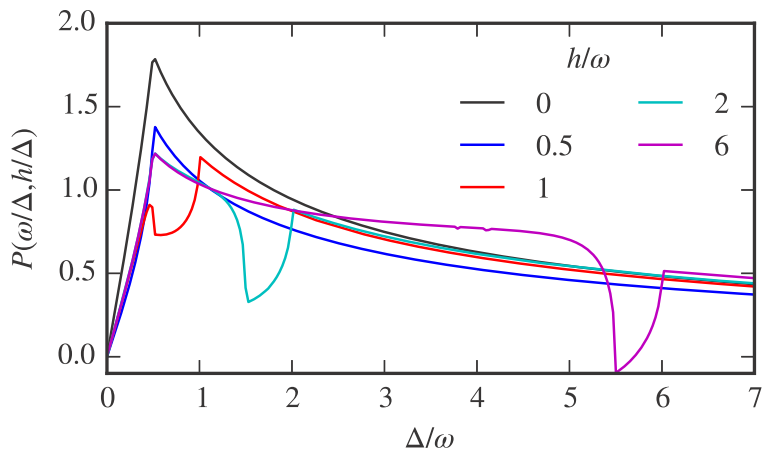

FIG. 4. Function $P$ for different exchange fields $h$. The resonant dips are located at $\Delta=h$.

function,

$$
\begin{aligned}
P(w, y)= & \int_{1}^{\infty} d x \frac{[Z(x, y)-Z(x+w, y)][x(x+w)+1]}{\sqrt{x^{2}-1} \sqrt{(x+w)^{2}-1}} \\
& +\theta(w-2) \int_{1}^{w-1} d x \frac{Z(x, y)[x(x-w)+1]}{\sqrt{x^{2}-1} \sqrt{(x-w)^{2}-1}} .
\end{aligned}
$$

Here,

$$
Z(x, y)=\frac{1}{2} \sum_{\alpha= \pm} \frac{\sum_{\gamma= \pm} F[x \Delta+(\alpha+\gamma) y \Delta]}{\sum_{\gamma= \pm} N[x \Delta+(\alpha+\gamma) y \Delta]} .
$$

In the absence of spin splitting, $Z(x, y=0)=1 / x$. The function $P$ is plotted in Fig. 4. We can also find its asymptotic behavior for $w \rightarrow 0$,

$$
P(w, y) \simeq \begin{cases}\sqrt{\frac{2 y w}{1+y}}+\frac{w \ln \frac{8}{w}}{2+2 y}, & w \ll y<1, \\ \sqrt{8 w}-\left(\ln \frac{8}{w}-1\right) w-\frac{w^{3 / 2}}{\sqrt{2}}, & w \ll 1 \ll y, \\ (\ln (8 / w)-1) w, & y \ll w \ll 1 .\end{cases}
$$

For $w \gg 1$, on the other hand,

$$
P(w, y) \simeq \begin{cases}\pi / w, & y \ll 1 \ll w, \\ 2 / w, & 1 \ll w, y ;|y-w| \gg 1 .\end{cases}
$$

These limits do not include the feature at $\Delta=h$.

As follows from the gap equation (24), close to $T=T_{c}$ the $\Delta(T)$ relation is determined by $T / T_{c}-1 \propto P(\Delta / \omega, h / \omega)$. The order parameter $\Delta(T)$ is then given by the curves in Fig. 4 , with the $y$ axis $\propto T-T_{c}$. Additional features in the $\Delta(T)$ relation may appear at $\Delta(T)=h$. In particular, the relation is multivalued around this point, indicating that we can expect discontinuous transitions as a function of temperature. This is related to changes in possible relaxation channels around $\Delta \sim h$ : for $\Delta(T)>h$ the spin-averaged density of states is gapped at $E<|\Delta|-|h|$, but for $\Delta(T)<h$ the averaged DOS is gapless, as the spin splitting is large enough to separate the energy gaps of the two spin species. The gap enhancement by microwave driving, however, continues to increase in the $\Delta(T)<h$ regime as long as $\omega<2 \Delta(T)$, and is larger at some frequencies than without spin splitting.

To obtain a more accurate picture, we can solve Eqs. (1) and (19) numerically. Such results are shown in Figs. 5 and 6. (a)

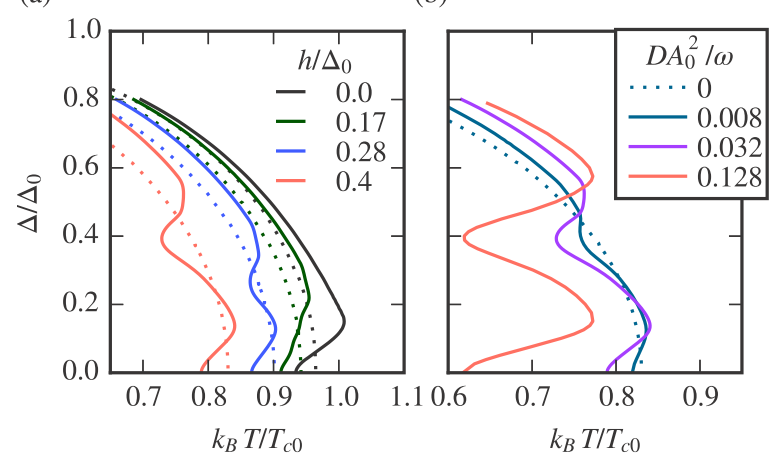

FIG. 5. Order parameter $\Delta$ for (a) different exchange fields for $D A_{0}^{2} / \omega=0.032$, and (b) different drive amplitudes for $h / \Delta_{0}=$ 0.4. Dotted lines correspond to $D A_{0}^{2}=0$. Spin-flip scattering time is $\tau_{\mathrm{sn}} T_{c 0}=12.5, \beta=0.5$ and $\alpha_{\mathrm{orb}}=0.01, \omega / \Delta_{0}=0.2$. Inelastic relaxation is described by a phonon model with $\tau_{\text {eph }, 0} T_{c 0}=100$.

Figure 5(a) indicates the appearance of a multivalued gap, when $h \gtrsim \omega$ and $\Delta \approx h$, as found analytically. From Fig. 6 we can note that the feature occurs for a wide range of scattering times. The feature is absent if there is no exchange field, and also if there is no spin-flip scattering, so that the qualitative conclusions based on Eq. (21) also apply at lower temperatures.

\section{DISCUSSION}

Microwave electric field in a spin-split superconductor drives the quasiparticles lying above the superconducting gap into a nonequilibrium state. In the absence of spinflipping processes, it, however, cannot generate charge or spin imbalance in the diffusive limit. Presence of spin-flip scattering enables generation of spin imbalance, and results to a photoelectric signal observable with ferromagnetic probes. The effect is closely related to the thermoelectric effects in magnetic superconductors. In addition, the excitation causes an instability in the superconducting order parameter when the energy gap becomes comparable to the exchange splitting of the spectrum.

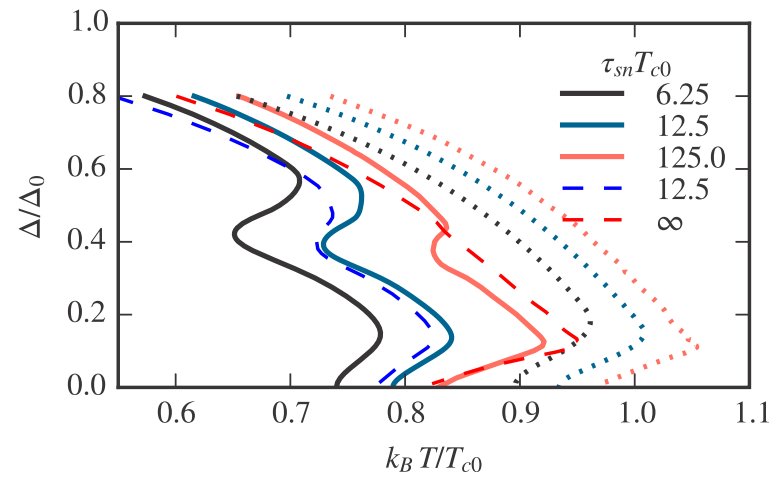

FIG. 6. Order parameter $\Delta$ for different spin-flip scattering times $\tau_{\mathrm{sn}}$. Here, $h / \Delta_{0}=0.4$, and other parameters are as in Fig. 5(a). The inelastic relaxation is modeled either via phonon model (solid lines) or relaxation time approximation (dashed). Results for $h=0$ are also shown (dotted) 
To conclude, we describe production of spin imbalance and a photo-spin-electric effect in spin-split superconductors, and present calculations in relevant parameter ranges. The effects are experimentally accessible with state-of-the art methods. A number of recent experiments in similar systems exist [5,8,9], including also microwave excitation in the $\mathrm{GHz}$ frequency range [9]. In addition to using ferromagnetic probes for detecting the photospin signal, the effect can also be seen indirectly by observing the discontinuous transitions in the superconducting order parameter. These are experimentally accessible via measurements of the tunneling DOS in the superconductor, or, for example, via measurement of the supercurrent.

\section{ACKNOWLEDGMENTS}

We thank M. Silaev for discussions. P.V. and T.T.H. acknowledge the Academy of Finland for financial support. T.T.H. acknowledges funding from the European Research Council (Grant No. 240362-Heattronics). The work of F.S.B. was supported by Spanish Ministerio de Economía y Competitividad (MINECO) through Project No. FIS2014-55987-P and Grupos Consolidados UPV/EHU del Gobierno Vasco (Grant No. IT-756-13).

\section{APPENDIX A: ELECTRON-PHONON INTERACTION}

We use a simplified model for inelastic relaxation due to electron-phonon interaction [27,39],

$$
\begin{aligned}
\hat{\sigma}^{R}(t)= & -g_{\mathrm{eph}}\left\{\tilde{D}^{R}(t) \hat{g}^{K}(t)+\tilde{D}^{K}(t) \hat{g}^{R}(t)\right\}, \\
\hat{\sigma}^{K}(t)= & -g_{\mathrm{eph}}\left\{\left[\tilde{D}^{R}(t)-\tilde{D}^{A}(t)\right]\left[\hat{g}^{R}(t)-\hat{g}^{A}(t)\right]\right. \\
& \left.+\tilde{D}^{K}(t) \hat{g}^{K}(t)\right\},
\end{aligned}
$$

where the Fourier transformed functions,

$$
\begin{gathered}
\tilde{D}^{R / A}(\omega)= \pm i \omega^{2} \operatorname{sgn} \omega, \\
\tilde{D}^{K}(\omega)=\left[\tilde{D}^{R}(\omega)-\tilde{D}^{A}(\omega)\right] \operatorname{coth} \frac{\omega}{2 T_{\mathrm{ph}}},
\end{gathered}
$$

arise from weighed Fermi surface averages of the phonon Green functions. Parts that do not contribute to the collision integral have been subtracted. The collision integral assumes a standard form,

$$
\begin{aligned}
\mathcal{I}_{L \sigma}= & g_{\mathrm{eph}} \int_{-\infty}^{\infty} \frac{d \omega}{8 \pi} \omega|\omega| \operatorname{tr}\left[\hat{g}^{\mathrm{RA}}(E) \hat{g}^{\mathrm{RA}}(E-\omega) s_{\sigma}\right] \\
& \times\left\{f_{L \sigma, E} f_{L \sigma, E-\omega}-1+\left[f_{L \sigma, E}-f_{L \sigma, E-\omega}\right] \operatorname{coth} \frac{\omega}{2 T_{\mathrm{ph}}}\right\},
\end{aligned}
$$

where $\hat{g}^{\mathrm{RA}}=\hat{g}^{R}-\hat{g}^{A}$. The prefactor $g_{\text {eph }}$ can be defined in terms of a relaxation rate,

$$
\tau_{\text {eph }, 0}^{-1}=4 \frac{7 \zeta(3) g_{\text {eph }}}{\pi} T_{c, 0}^{3},
$$

at temperature $T_{c, 0}$ in the normal state at the Fermi surface, where $\hat{\sigma}^{R}=-i \hat{\tau}_{3} /\left(2 \tau_{\mathrm{eph}, 0}\right)$.

\section{APPENDIX B: NUMERICAL DETAILS}

We solve Eq. (1) numerically via a Jacobian-free NewtonGMRES method [40]. To obtain a preconditioner, we use automatic differentiation to compute the Jacobian of the energylocal terms, excluding the self-energy parts $\left[\check{\sigma}_{\text {eph }}+\check{\sigma}_{A}, \breve{g}\right]$. The energy convolutions in the self-energies, after energy discretization, are computed via fast Fourier transforms.
[1] J. Linder and J. W. A. Robinson, Nat. Phys. 11, 307 (2015).

[2] S. A. Kivelson and D. S. Rokhsar, Phys. Rev. B 41, 11693 (1990).

[3] H. L. Zhao and S. Hershfield, Phys. Rev. B 52, 3632 (1995).

[4] C. H. L. Quay, D. Chevallier, C. Bena, and M. Aprili, Nat. Phys. 9, 84 (2013).

[5] F. Hübler, M. J. Wolf, D. Beckmann, and H. v. Löhneysen, Phys. Rev. Lett. 109, 207001 (2012).

[6] M. J. Wolf, F. Hübler, S. Kolenda, H. v. Löhneysen, and D. Beckmann, Phys. Rev. B 87, 024517 (2013).

[7] M. J. Wolf, C. Sürgers, G. Fischer, and D. Beckmann, Phys. Rev. B 90, 144509 (2014).

[8] C. H. L. Quay, C. Dutreix, D. Chevallier, C. Bena, and M. Aprili, arXiv:1408.1832.

[9] C. H. L. Quay, M. Weideneder, Y. Chiffaudel, C. Strunk, and M. Aprili, Nature Communications 6, 8660 (2015).

[10] P. M. Tedrow and R. Meservey, Phys. Rev. Lett. 26, 192 (1971).

[11] P. M. Tedrow, J. E. Tkaczyk, and A. Kumar, Phys. Rev. Lett. 56, 1746 (1986).

[12] M. Silaev, P. Virtanen, F. S. Bergeret, and T. T. Heikkilä, Phys. Rev. Lett. 114, 167002 (2015).
[13] I. V. Bobkova and A. M. Bobkov, Pis'ma Zh. Eksp. Teor. Fiz. 101, 124 (2015).

[14] T. Krishtop, M. Houzet, and J. S. Meyer, Phys. Rev. B 91, 121407 (2015).

[15] M. S. Kalenkov, A. D. Zaikin, and L. S. Kuzmin, Phys. Rev. Lett. 109, 147004 (2012).

[16] P. Machon, M. Eschrig, and W. Belzig, Phys. Rev. Lett. 110, 047002 (2013).

[17] M. S. Kalenkov and A. D. Zaikin, Phys. Rev. B 90, 134502 (2014).

[18] P. Machon, M. Eschrig, and W. Belzig, New J. Phys. 16, 073002 (2014).

[19] A. Ozaeta, P. Virtanen, F. S. Bergeret, and T. T. Heikkilä, Phys. Rev. Lett. 112, 057001 (2014).

[20] S. Kolenda, M. J. Wolf, and D. Beckmann, arXiv:1509.05568.

[21] A. V. Zaitsev, Sov. Phys. JETP 63, 579 (1986).

[22] M. S. Kalenkov and A. D. Zaikin, Phys. Rev. B 92, 014507 (2015).

[23] G. M. Eliashberg, JETP Lett. 11, 114 (1970).

[24] G.-X. Miao and J. S. Moodera, Phys. Chem. Chem. Phys. 17, 751 (2015).

[25] F. S. Bergeret, A. F. Volkov, and K. B. Efetov, Rev. Mod. Phys. 77, 1321 (2005). 
[26] K. D. Usadel, Phys. Rev. Lett. 25, 507 (1970).

[27] N. B. Kopnin, Theory of Nonequilibrium Superconductivity, International Series of Monographs on Physics, No. 110 (Oxford University Press, Oxford, 2001).

[28] J. P. Morten, A. Brataas, and W. Belzig, Phys. Rev. B 70, 212508 (2004).

[29] J. P. Morten, A. Brataas, and W. Belzig, Phys. Rev. B 72, 014510 (2005).

[30] N. Poli, J. P. Morten, M. Urech, A. Brataas, D. B. Haviland, and V. Korenivski, Phys. Rev. Lett. 100, 136601 (2008).

[31] W. Belzig, C. Bruder, and G. Schön, Phys. Rev. B 54, 9443 (1996).
[32] F. S. Bergeret, A. Verso, and A. F. Volkov, Phys. Rev. B 86, 214516 (2012).

[33] P. K. Tien and J. P. Gordon, Phys. Rev. 129, 647 (1963).

[34] J. Tucker and M. Feldman, Rev. Mod. Phys. 57, 1055 (1985).

[35] R. E. Horstman and J. Wolter, Phys. Lett. A 82, 43 (1981).

[36] J. E. Mooij and T. M. Klapwijk, Phys. Rev. B 27, 3054 (1983).

[37] K. Maki and T. Tsuneto, Prog. Theor. Phys. 31, 945 (1964).

[38] K. Maki, Prog. Theor. Phys. 32, 29 (1964).

[39] G. M. Eliashberg, Sov. Phys. JETP 34, 668 (1972).

[40] D. Knoll and D. Keyes, J. Comp. Phys. 193, 357 (2003). 